\title{
Los escarabajos estercoleros (Coleoptera: Scarabaeidae: Scarabaeinae) del Bosque Protector Oglán Alto, Pastaza, Ecuador
}

\section{The dung beetles (Coleoptera: Scarabaeidae: Scarabaeinae) from Oglán Alto Protective Forest, Pastaza, Ecuador}

\author{
William R. Chamorro, Freddy O. Gallo, Soraya Delgado, Sandra I. Enríquez, Verónica Guasumba
} y Germán López-Iborra

\section{Resumen}

El Bosque Protector Oglán Alto tiene una extensión de 3100 hectáreas y está dominado por bosques de tierras bajas y bosques de piedemonte amazónicos que albergan fauna y flora de gran diversidad; sin embargo, esta ha sido pobremente estudiada, especialmente el grupo de los insectos. Presentamos el inventario de los escarabajos estercoleros (Coleoptera: Scarabaeidae: Scarabaeinae) en esta localidad. Un total de 10,627 individuos pertenecientes a 17 géneros y 65 especies fueron determinados durante 18 meses de recolección en la que se utilizaron 11 técnicas de muestreo. Esta investigación presenta un listado actualizado, acompañado de una guía fotográfica para los principales géneros y especies, la cual servirá para la fácil identificación de este grupo de escarabajos en la región.

Palabras clave. Bosques amazónicos. Grupo funcional. Inventario de especies. Técnicas de muestreo.

\begin{abstract}
The Oglán Alto Protective Forest has an area of 3100 hectares and is dominated by Amazonian lowland and piedmont forests, which harbor great diversity of fauna and flora. However, such diversity has been poorly studied, especially insects. Here we present an inventory of the dung beetles (Coleoptera: Scarabaeidae: Scarabaeinae) for this locality. A total of 10,627 individuals, belonging to 17 genera and 65 species, were determined during 18 months of collection, in which 11 different sampling methods were used. This research presents and updated checklist and a photographic guide of the main genera and species of dung beetles identified in the study area.
\end{abstract}

Keywords. Amazonian forests. Collecting techniques. Functional group. Species inventory. 


\section{Introducción}

Los escarabajos copronecrófagos, comúnmente llamados estercoleros, pertenecen a la subfamilia Scarabaeinae. Son muy utilizados a nivel mundial en estudios de biodiversidad, conservación y ecología, especialmente porque son un grupo de insectos de fácil recolección e identificación a nivel de géneros, y en algunos casos, hasta especie. Además, están distribuidos en casi todos los biomas naturales, cuentan con suficiente bibliografía especializada y el costo de muestreo resulta bastante económico (Favila \& Halffter, 1997).

En Ecuador, pocos son los estudios que tratan sobre taxonomía y biodiversidad a través de inventarios locales para este grupo de escarabajos. Vale mencionar los siguientes estudios en las localidades de Río Palenque-Los Ríos (Peck \& Forsyth, 1982), Otonga-Cotopaxí (Enríquez \& Onore, 2001), Ángel Rouby y Untsuants cordillera del Kutuku-Morona Santiago (Celi et al., 2004), senderos de la estación Oglán-Pastaza (Carvajal \& Villamarín, 2007), Chiruisla-Orellana (Carpio et al., 2009), Alamala-Loja (Domínguez et al., 2015) y el gradiente sobre la estribación oriental de los Andes, desde Papallacta a Misahuallí-Napo (Espinoza \& Noriega, 2018).

El Bosque Protector Oglán Alto está ubicado en la provincia amazónica de Pastaza. El bosque tiene una extensión de 3100 hectáreas, en las que viven las comunidades indígenas de pueblos kichwas y shuar y tiene una gran riqueza de flora, fauna, tipos de bosque y formaciones vegetales (Palacios et al., 1999; Cerón et al., 2007). Desafortunadamente, dentro de esta área protegida se encuentran pozos petroleros que el Estado ecuatoriano piensa explotar dentro de algunos años, afectando así la biodiversidad. El objetivo de este trabajo fue inventariar la fauna de escarabajos estercoleros (Coleoptera: Scarabaeinae) existente en esta localidad, utilizando diferentes métodos de recolección.

\section{Materiales y métodos}

Área de estudio. Realizamos este estudio en los remanentes secundarios del Bosque Protector Oglán Alto, pertene- cientes a las comunidades Kichwa Pablo López y Shuar Washents, localizadas cerca al río Oglán y al poblado de Arajuno en la provincia de Pastaza, Ecuador (1019'29" N, $77^{\circ} 41^{\prime} 20^{\prime \prime} \mathrm{O}$ ). La temperatura varía entre 16 y $30^{\circ} \mathrm{C}$ y el promedio anual de lluvias excede los $4000 \mathrm{~mm}$ según datos de Palacios \& Ontaneda (2009). De acuerdo con Palacios et al. (1999), el área de estudio está cubierta especialmente por dos formaciones vegetales: bosques siempreverdes de tierras bajas (tierra firme) y bosques siempre-verdes de piedemonte. Finalmente, según Cerón et al. (2007), los tipos de paisajes que presenta este bosque protector son: valles aluviales, bosques de colinas, bosques ribereños y chacras o zonas de cultivo. El gradiente altitudinal de muestreo fue desde 540 hasta 950 m s. n. m. (Figura 1).

Fase de campo. El inventario se realizó con la aplicación de 11 técnicas de recolección, entre métodos activos, pasivos, diurnos y nocturnos (Figura 1).

A continuación, se describen cada uno de los métodos de recolección y se resalta el periodo de muestreo:

a) Recolección manual: Esta técnica permitió capturar los escarabajos estercoleros en diferentes sustratos, principalmente en hojas y ramas de los senderos y bosques. Una vez capturados, los colocamos en un frasco mortal que contenía corcho molido y acetato de etilo. Aplicamos esta técnica durante una hora, el quinto día de muestreo de la última semana de cada mes, desde marzo del 2008 hasta agosto del 2009.

b) Fumigación del dosel: Fumigamos en bosques de valles aluviales, bosques ribereños y bosques colinados en los meses de julio, agosto y septiembre del 2008 y abril y julio del 2009. Utilizamos la metodología propuesta por Erwin (1989), colocando un promedio de diez sabanas de $9 \mathrm{~m}^{2}$ bajo los estratos arbóreos. Al anochecer fumigamos las copas de los árboles por un periodo de un minuto. Para este proceso se utilizó un insecticida biodegradable (Permetrina Técnica al 3\%), aplicado con una fumigadora Golden Dyna Fogger. A la mañana siguiente se recogieron los escarabajos de las sábanas y se colocaron en frascos con alcohol al $75 \%$. Esta técnica permitió capturar algunos escarabajos coprófagos que habitan principalmente en el dosel. 


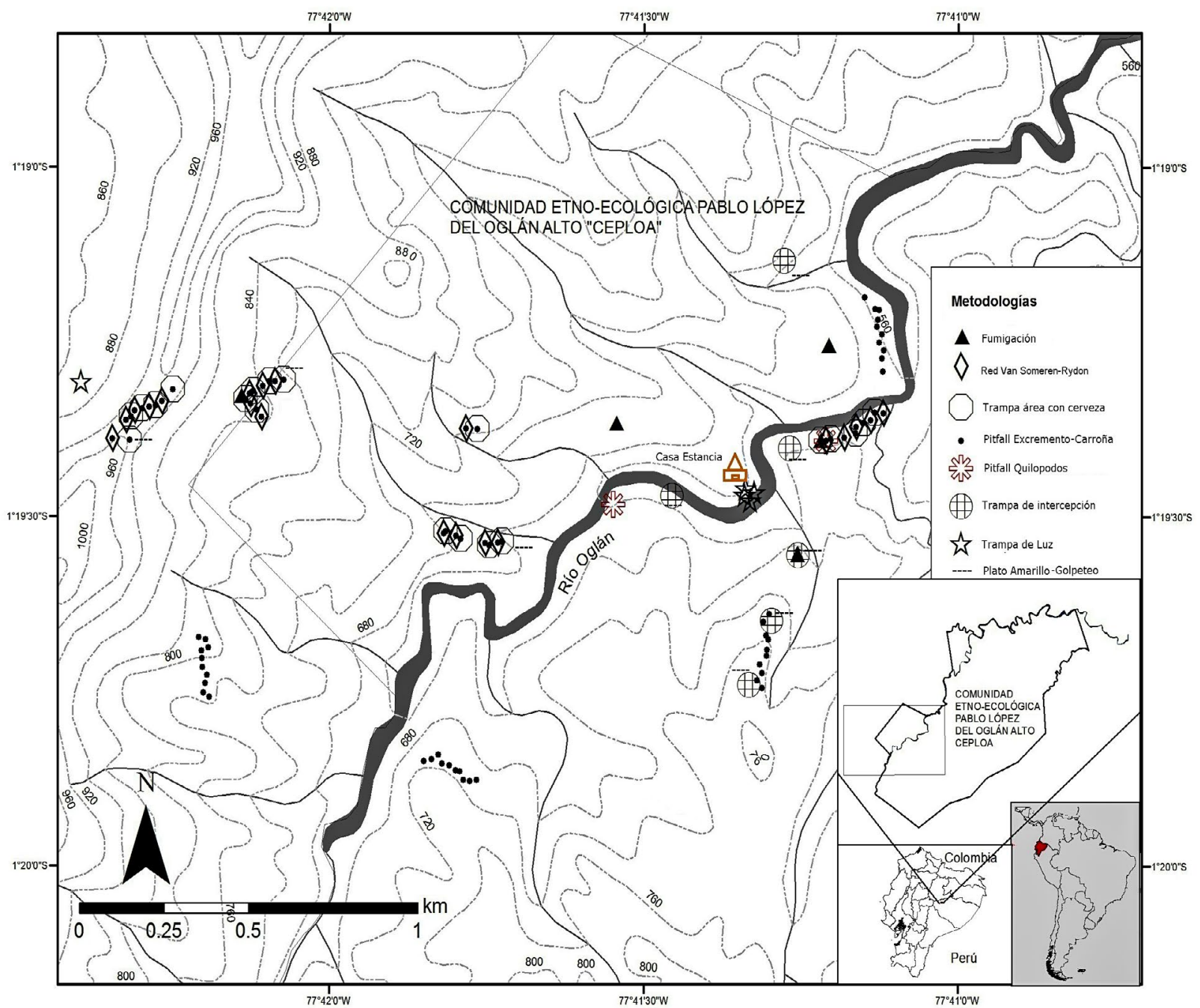

Figura 1. Área de estudio del Bosque Protector Oglán Alto, Pastaza, Ecuador, y metodologías utilizadas para el inventario de escarabajos coprófagos de la zona.

c) Golpeteo: Esta técnica es utilizada para capturar escarabajos que están asociados a vegetación arbórea y arbustiva especialmente de bosques colinados, ribereños y de valles aluviales. Utilizamos una sábana de golpeteo de $1 \mathrm{~m}^{2}$ de tela blanca, con esquinas reforzadas a manera de bolsillos en los que se insertaron dos tubos flexibles, lo que permitió mantener la sábana extendida para posteriormente colocarla debajo de las ramas o arbustos que contenían hojas secas. Con la ayuda de una vara delgada, se procedió a golpear y sacudir las ramas para que los escarabajos cayeran sobre la sába- na. Esta técnica se efectuó el quinto día de muestreo de la última semana de cada mes, desde marzo del 2008 hasta agosto del 2009, durante una hora.

d) Platos amarillos: Utilizamos platos de $68 \mathrm{~cm}$ de diámetro por $3 \mathrm{~cm}$ de profundidad, que contenían una mezcla de agua, detergente sin fragancia y una porción de sal para eliminar la tensión superficial del líquido. Los platos fueron ubicados durante 24 horas en bosques colinados y de valles aluviales, durante la última semana de cada mes desde febrero hasta agosto del 2009. 
e) Red de neblina: Capturamos escarabajos estercoleros en redes de neblina de 12 y $9 \mathrm{~m}$ de largo. Las redes fueron ubicadas generalmente en bosques colinados y bosques de valles aluviales. Las recolecciones se llevaron a cabo en los meses de abril, mayo y junio del 2009.

f) Trampas áreas: Realizamos cuatro transectos lineales de $200 \mathrm{~m}$ de longitud, ubicados a lo largo de bosques colinados y valles aluviales sobre un gradiente altitudinal (transecto 1: $947 \mathrm{~m} \mathrm{~s}$. n. m.; transecto 2: $820 \mathrm{~m} \mathrm{~s}$. n. m.; transecto 3: $640 \mathrm{~m} \mathrm{s.} \mathrm{n.} \mathrm{m.;} \mathrm{transecto} \mathrm{4:} 577 \mathrm{~m} \mathrm{~s}$. n. $\mathrm{m}$.). En cada transecto las trampas se colgaron de árboles aproximadamente a $6 \mathrm{~m}$ del suelo, separadas entre sí por $22 \mathrm{~m}$ de distancia. En cada trampa colocamos como cebo una mezcla de cerveza con panela molida, levadura y jugo de mango. La trampa se fabricó con una botella de plástico de 3 litros cortada en dos, en la cual la parte superior se volteó y se colocó a manera de embudo. Los insectos atraídos por el cebo entraron en el embudo formado por el cuello de la botella y luego no podían escapar. La actividad de estas trampas fue de 6 días, el periodo de muestreo comprendió desde marzo del 2008 hasta agosto del 2009, y se recolectó generalmente la última semana de cada mes.

g) Trampa de intercepción: Utilizamos una malla de tela fina de color negro, de forma rectangular, con medidas de $1.8 \mathrm{~m}$ de ancho y $1.5 \mathrm{~m}$ de alto. La tela tenía un pliegue a lo largo de cada lado, por donde se introdujeron un par de tubos cilíndricos y delgados que se clavaron al suelo. Con la ayuda de cuerdas, la malla se colocó estirada en posición horizontal sobre cuatro bandejas rectangulares de metal, de poca profundidad. A estos recipientes les añadimos una mezcla de agua saturada de sal y detergente sin fragancia; adicionalmente colocamos un techo de plástico que protegía la malla y los recipientes de la lluvia. Estas trampas fueron ubicadas durante 24 horas en bosques colinados y de valles aluviales, durante la última semana de cada mes, desde febrero hasta agosto del 2009.

h) Trampa de luz: Esta técnica consistió en una sábana blanca de $3 \times 3 \mathrm{~m}$, extendida horizontalmente con un foco de vapor de mercurio de 400 watts generalmente ubicado frente o encima de la sábana y que funcionó con un generador a gasolina. Esta trampa operó por cuatro horas durante seis noches de la última semana de cada mes, coincidiendo con la fase de luna nueva. Todos los ejemplares capturados fueron sacrificados en frascos con acetato de etilo y luego dispuestos en mantas de algodón para su transporte al laboratorio. Generalmente se ubicó esta metodología en zonas abiertas y chacras, desde marzo del 2008 hasta agosto del 2009.

i) Trampas de caída tipo "pitfall" con excremento humano o carroña: Este método de muestreo se utilizó en los mismos cuatro transectos altitudinales de las trampas aéreas y posteriormente con cuatro transectos en otro sitio (con altitudes similares al primero). En cada transecto se colocaron 20 trampas dispuestas cada 22 metros, 10 con excremento humano y 10 con carroña (pescado descompuesto proveniente del río Oglán). En cada punto medido colocamos una trampa con excremento humano y, separada por $4 \mathrm{~m}$, una trampa con carroña. Se utilizaron tarrinas de plástico de $12 \mathrm{~cm}$ de diámetro por $14 \mathrm{~cm}$ de profundidad, con dos aberturas a los lados superiores, para la entrada de los escarabajos y una tapa para protección de las lluvias. La actividad de las trampas en cada transecto fue de 48 horas. Cada recipiente tenía una mezcla de agua saturada de sal y detergente sin fragancia, con el cebo dentro de una gasa y suspendido encima de la trampa. Los escarabajos fueron recolectados y conservados en alcohol al $75 \%$, para su posterior traslado al laboratorio. El periodo de muestreo comprendió desde marzo del 2008 hasta agosto del 2009 y se recolectó generalmente la última semana de cada mes.

j) Trampas de caída tipo "pitfall" cebadas con miriápodos muertos: Utilizamos cinco trampas pitfall cebadas con diplópodos y quilópodos en proceso de descomposición, ubicadas generalmente en bosques de valles aluviales; cada trampa contenía una mezcla de agua saturada de sal y detergente sin fragancia, con el miriápodo dentro de una gasa y suspendido 
encima de la trampa. El periodo de muestreo fue en los meses de septiembre y octubre del 2008 y en los meses de enero y julio del 2009.

k) Redes aéreas Van Someren-Rydon. Fueron dispuestas cada $25 \mathrm{~m}$ y colocadas alternadamente a $1.5 \mathrm{~m}$ en el sotobosque y a $15 \mathrm{~m}$ de altura, desde el suelo al nivel del dosel. Cada red fue cebada con plátano maduro fermentado y pescado podrido. Estas trampas fueron ubicadas en bosques de colina y de valles aluviales, permaneciendo activas durante 48 horas generalmente la última semana de cada mes desde febrero hasta agosto del 2009.

Identificación y preservación de especímenes. La mayoría del material recolectado fue conservado en alcohol al $75 \%$, posteriormente fue curado y algunos especímenes se montaron en alfileres entomológicos, con sus respectivas etiquetas de información. Algunos especímenes fueron preservados en acetato de etilo y colocados en mantas de algodón, principalmente para conservar su coloración y extraer la genitalia. Depositamos los especímenes montados en MGO-UCE (Museo Laboratorio Gustavo Orces, Universidad Central del Ecuador) con copias de duplicados en MECN (Museo Ecuatoriano de Ciencias Naturales) y CEMT (Setor de Entomologia da Coleção Zoológica da Universidade Federal de Mato Grosso Cuiabá). Para la identificación del respectivo material utilizamos literatura especializada (Génier, 1996, 2009; Cook, 1998, 2002; Ratcliffe \& Smith, 1999; Arnaud, 2002; Edmonds \& Zidek, 2004, 2010, 2012; Canhedo, 2006; Vaz-de-Mello et al., 2011; Silva et al., 2015) y contamos con la colaboración de especialistas para la validación final del listado. Finalmente se estableció el grupo funcional trófico de acuerdo con Halffter \& Edmonds (1982).

\section{Resultados}

Diversidad. Registramos un total de 10,627 individuos, pertenecientes a 65 especies y 18 géneros de
Scarabaeinae (Coleoptera: Scarabaeidae). El $66.15 \%$ de las especies fueron determinadas (43 en total), mientras que el 35.38 \% aún están como aff., ó a nivel de morofoespecie (sp.). El género Eurysternus fue el que registró mayor número de especies, nueve en total (ver Anexo 1). Las especies más abundantes (que sobrepasaron los 1000 individuos recolectados) son: Deltochilum crenulipes (Figura 4A); Dichotomius quinquelobatus (Figura 5A) y Eurysternus caribaeus (Figura 5D). Las especies más raras (con apenas un individuo recolectado) son: Ateuchus sp.; Bdelyrus lobatus (Figura 2D); Canthidium sp. 3; Dichotomius compressicollis (Figura 4E); Dichotomius sp. (Figura 5B); Phanaeus cambeforti (Figura 7D); Phanaeus haroldi (Figura 7F) y Scybalocanthon sp. Además registramos la presencia del género Canthonella y 31 especies que no fueron listadas por Carvajal \& Villamarín (2007).

Las trampas pitfall cebadas con excremento humano fueron las que obtuvieron un mayor número de registros (59 especies), seguidas por las trampas pitfall cebadas con carroña (41), trampas o redes de intercepción de vuelo (39) y la técnica de recolección manual (25). Las técnicas de muestreo de trampas pitfall con excremento humano, trampas pitfall con carroña, recolección manual, trampas áereas cebadas con cerveza y trampa de luz fueron los únicos métodos estandarizados y utilizados durante los 18 meses de muestreo.

Gremios tróficos. El comportamiento de nidificación y alimentación que presenta la comunidad de escarabajos estercoleros en Oglán están compuestos por tres gremios tróficos o grupos funcionales de los definidos por Halffter \& Edmonds (1982): escarabajos cavadores o paracópridos, con 35 especies (59.92\%, generalmente especies de tamaño grande y mediano); escarabajos rodadores o telecópridos, con 17 especies ( $29.23 \%$, generalmente especies de tamaño mediano y pequeño); y escarabajos moradores o endocópridos con 9 especies $(13.85 \%$, generalmente especies de tamaño mediano y pequeño). 
A

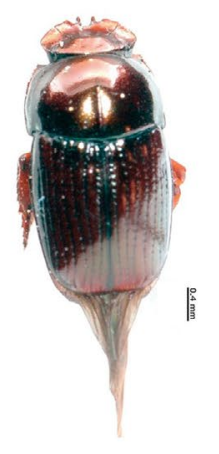

C

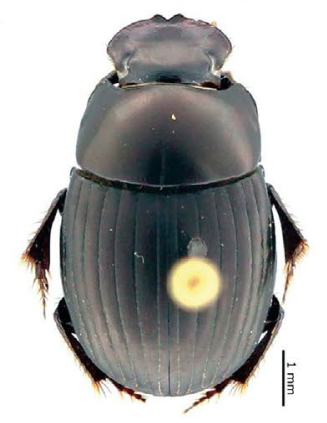

E

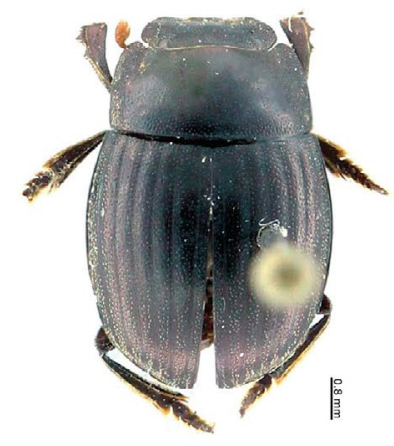

G

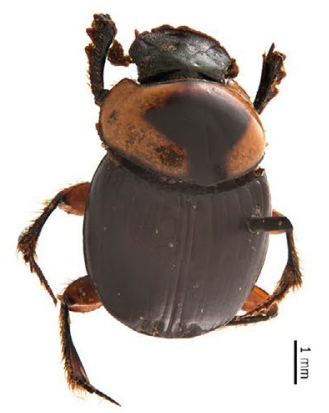

B

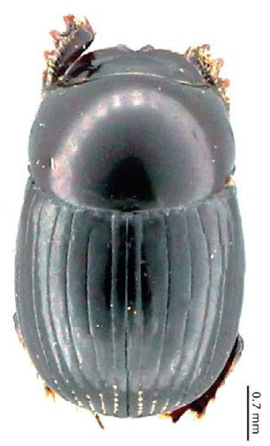

D

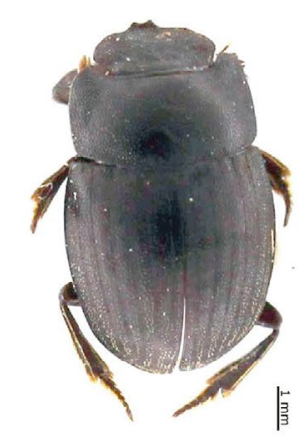

F

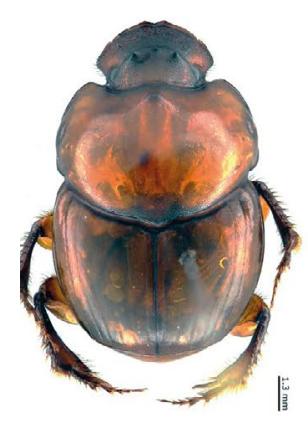

H

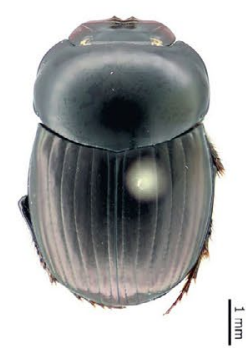

Figura 2. Especies registradas en el Bosque Protector Oglán Alto, Pastaza, Ecuador. A. Anomiopus aff. foveicollis, B. Ateuchus scatimoides, C. Ateuchus connexus, D. Bdelyrus genieri, E. Bdelyrus lobatus, F. Canthidium onitoides, G. Canthidium aff. gerstackeri, H. Canthidium (Neocanthidium) sp. 1. Fotografías: William R. Chamorro (A-F y H) y Colección de Insectos Universidad Particular de Loja (G). 
A

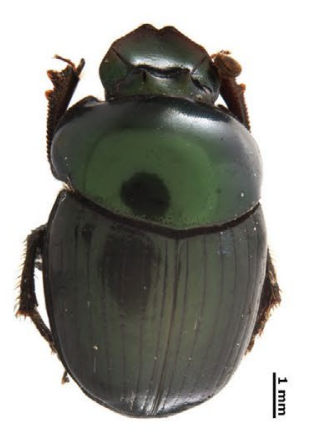

C

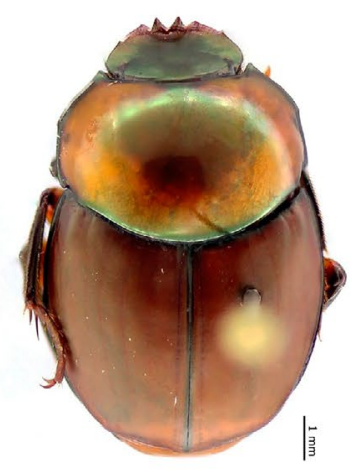

E

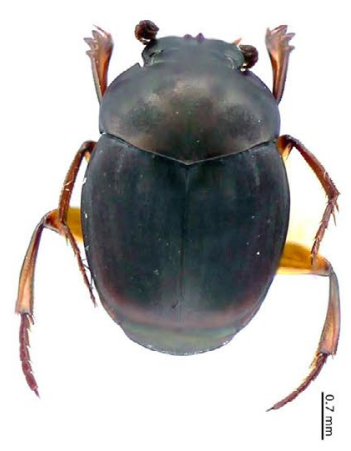

G

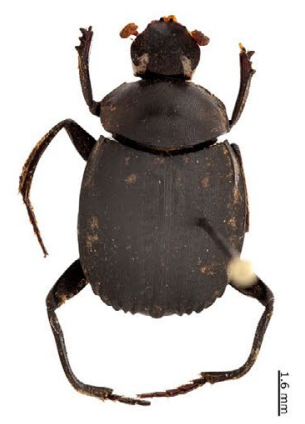

B

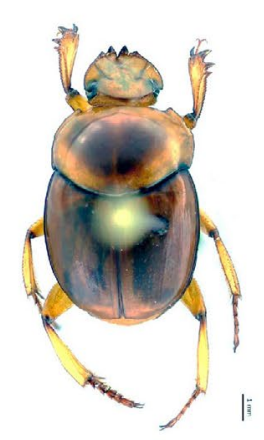

D

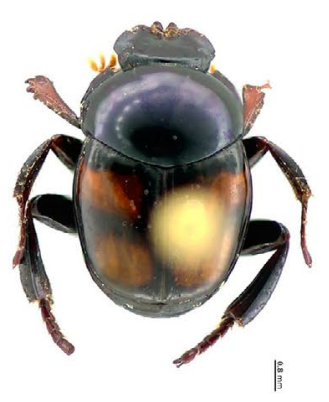

F

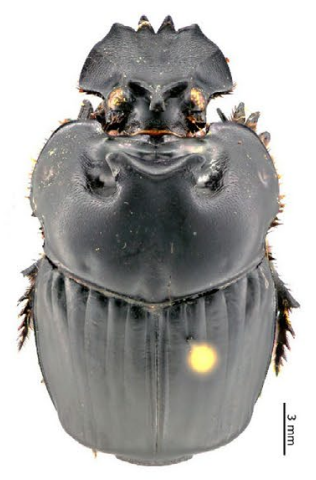

(H)

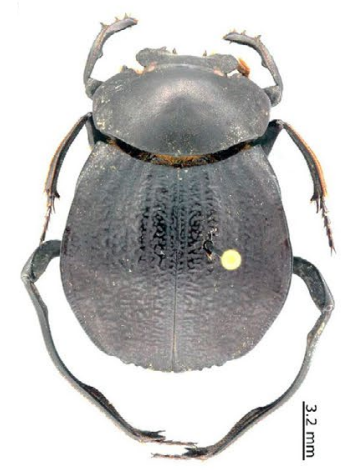

Figura 3. Especies registradas en el Bosque Protector Oglán Alto, Pastaza, Ecuador. A. Canthidium (Neocanthidium) sp. 2, B. Canthon ohausi, C. Canthon luteicollis, E. Canthon quadriguttatus, D. Canthon sericatus, G. Coprophanaeus telamon, macho, F. Deltochilum larseni, macho, H. Deltochilum orbiculare macho. Fotografías: Colección de Insectos Universidad Particular de Loja (A) y William R. Chamorro (B-H). 
A

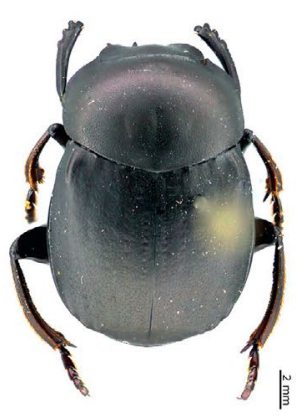

C

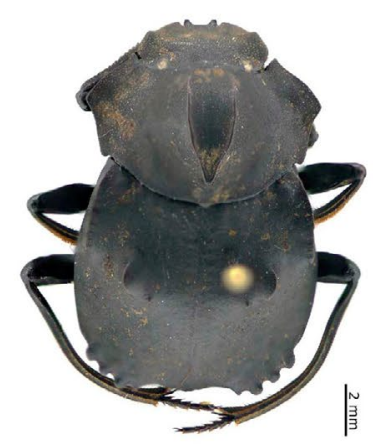

E

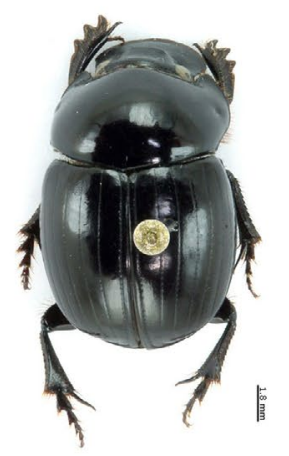

G

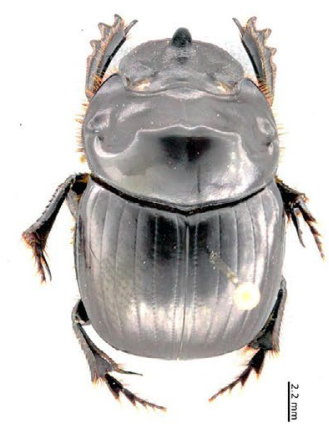

B

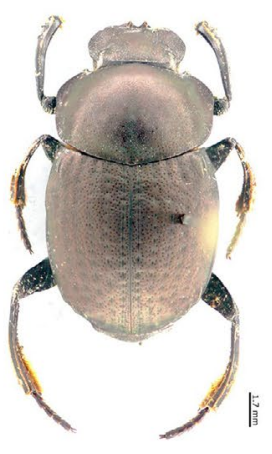

D

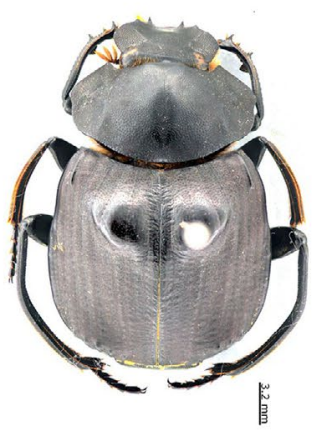

$\boldsymbol{F}$

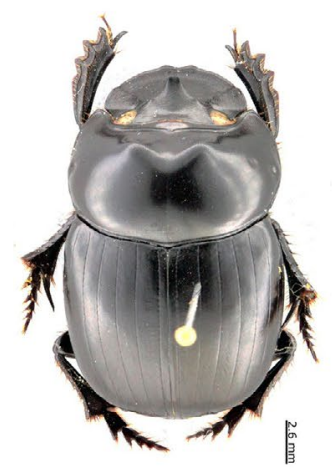

$\boldsymbol{H}$

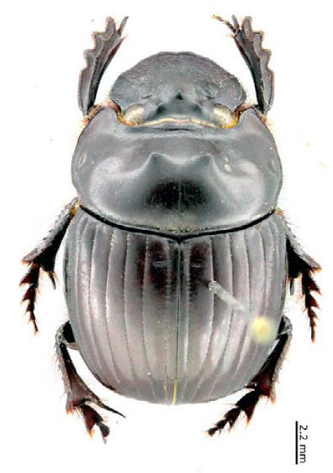

Figura 4. Especies registradas en el Bosque Protector Oglán Alto, Pastaza, Ecuador. A. Deltochilum crenulipes, macho. B. Deltochilum barbipes, C. Deltochilum carinatum, D. Deltochilum orbignyi amazonicum, macho. E. Dichotomius compressicollis, hembra. F. Dichotomius mamillatus, macho. G. Dichotomius ohausi, macho. H. Dichotomius podalirius. Fotografías: William R. Chamorro. 
A

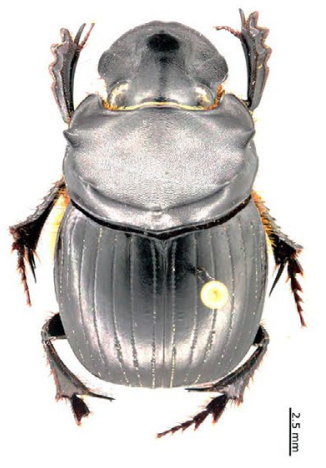

C

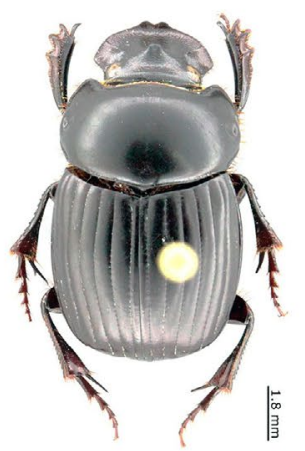

E

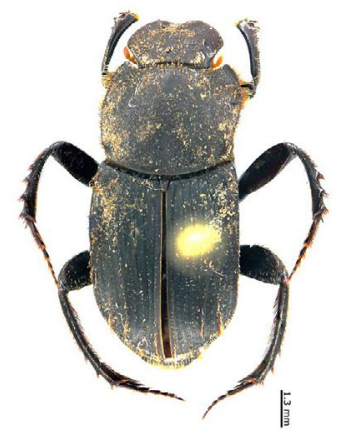

G

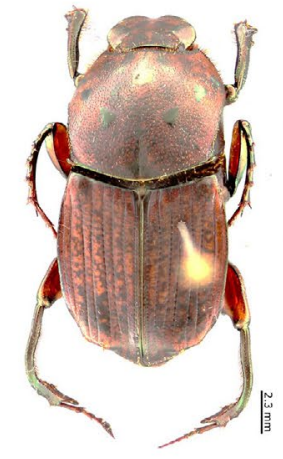

B

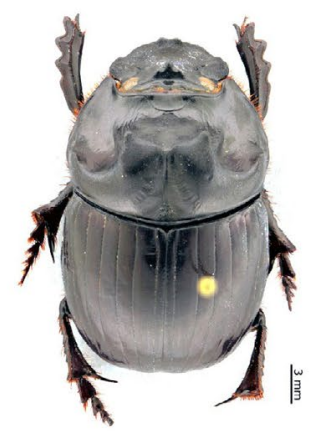

D

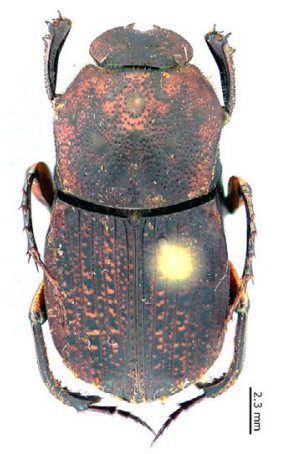

F

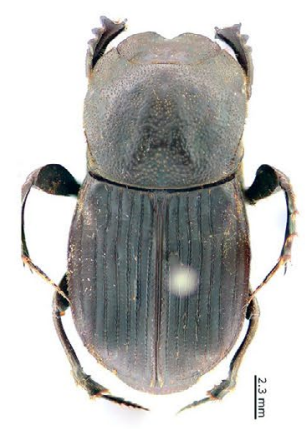

H

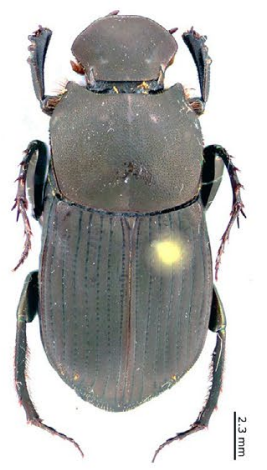

Figura 5. Especies registradas en el Bosque Protector Oglán Alto, Pastaza, Ecuador. A. Dichotomius quinquelobatus, macho, B. Dichotomius sp., C. Dichotomius aff. problematicus, D. Eurysternus caribaeus, E. Eurysternus cayennensis, F. Eurysternus foedus, G. Eurysternus hamaticollis, H. Eurysternus hypocrita. Fotografías: William R. Chamorro. 
(A)

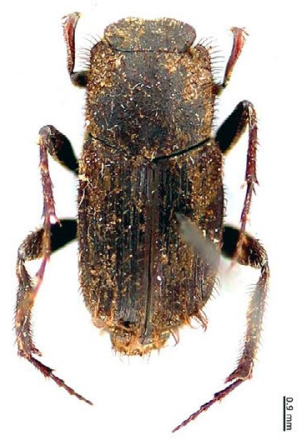

C

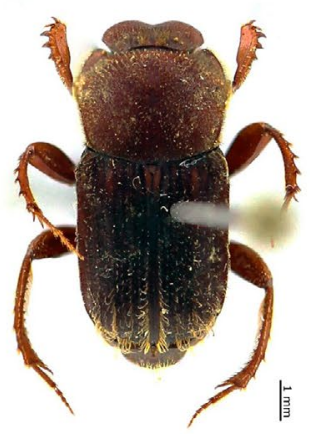

E

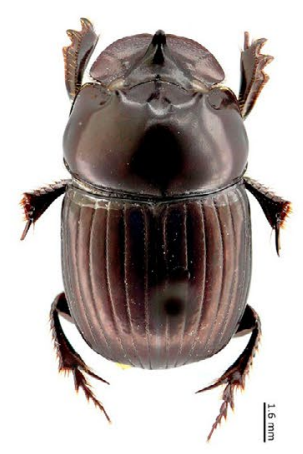

G

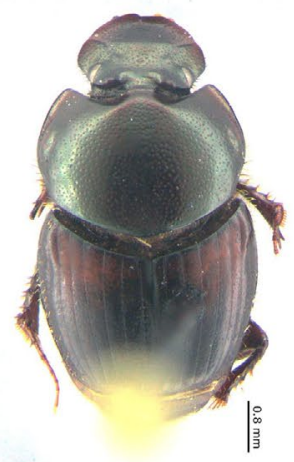

B

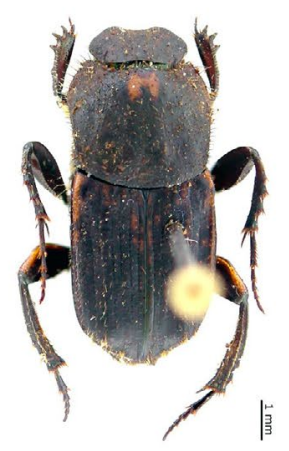

D

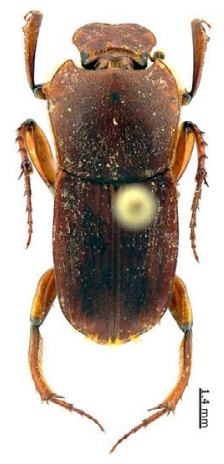

F

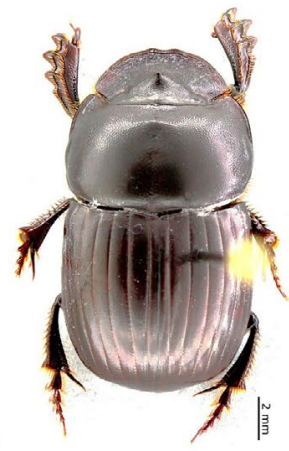

(H)

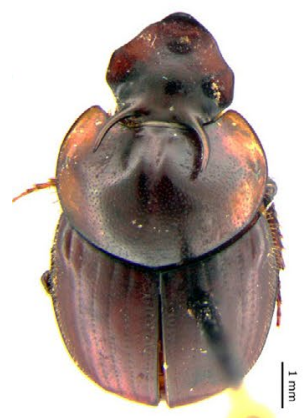

Figura 6. Especies registradas en el Bosque Protector Oglán Alto, Pastaza, Ecuador. A. Eurysternus lanuginosus, B. Eurysternus plebejus, C. Eurysternus vastiorum, D. Eurysternus wittmerorum, E. Ontherus diabolicus, macho, F. Ontherus pubens, G. Onthophagus rubrescens, macho, H. Onthophagus xanthomerus, macho, Fotografías: William R. Chamorro. 
A

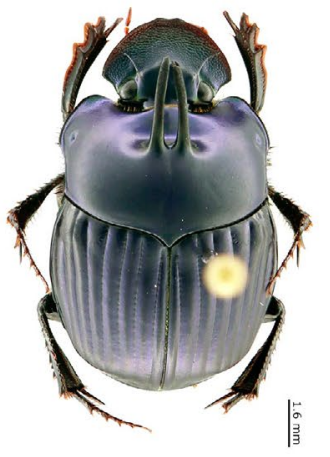

C

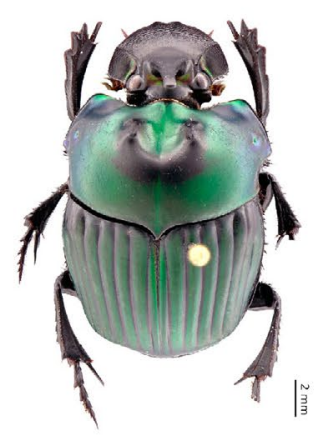

E

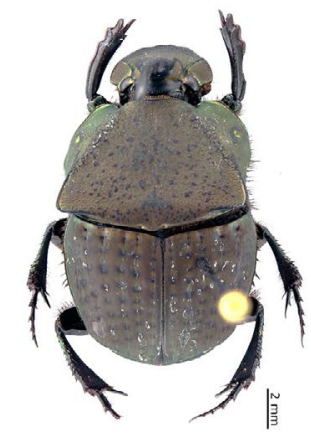

G

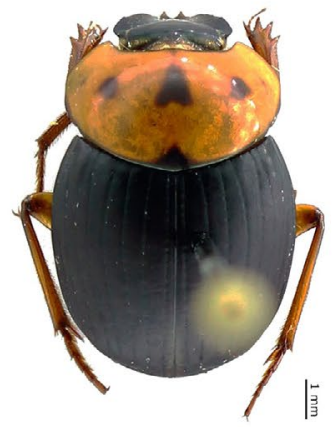

B

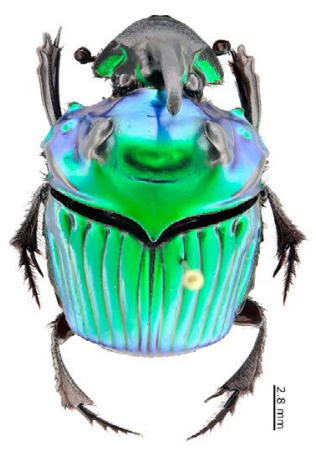

D

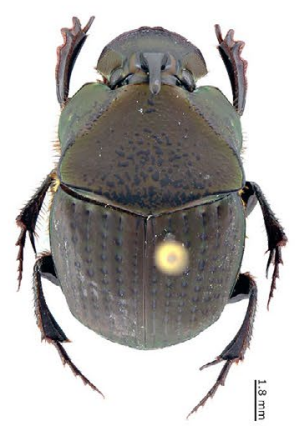

F

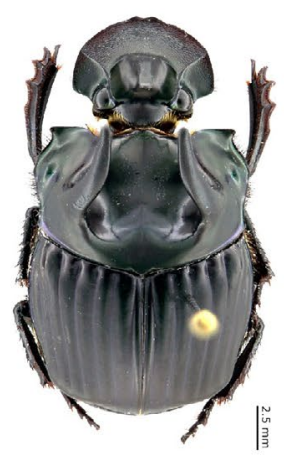

H

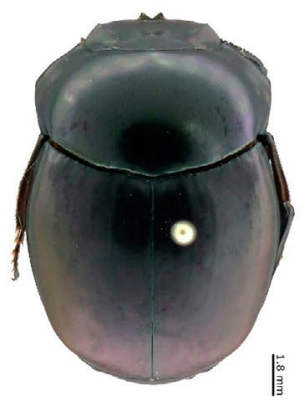

Figura 7. Especies registradas en el Bosque Protector Oglán Alto, Pastaza, Ecuador. A. Oxysternon spiniferum, macho, B. Oxysternon conspicillatum macho, C. Oxysternon silenus smaragdinum, macho, D. Phanaeus cambeforti, macho, E. Phanaeus chalcomelas macho, F. Phanaeus haroldi, macho, G. Scybalocanthon kaestneri, H. Sylvicanthon proseni. Fotografías: William R. Chamorro. 


\section{Discusión}

Diversidad. Ecuador registra un total de 33 géneros y 223 especies de escarabajos copronecrófagos Scarabaeinae (Coleoptera: Scarabaeidae), entre ellos 69 especies para la provincia amazónica de Pastaza. El Bosque Protector Oglán Alto alberga el 19.17 \% (teniendo en cuenta solamente especies identificadas) de esta riqueza de especies (Chamorro et al., 2018). Sin embargo, de las 45 especies endémicas conocidas para Ecuador (Chamorro et al., 2019), solo 2 especies se encontraron en esta localidad: Bdelyrus genieri Cook, 1998 (Figura 2D) y Scybalocanthon kaestneri (Balthasar, 1939) (Figura 7G) (Cook, 1998; Bezdek \& Hajek, 2011; Krajcik, 2012).

En la Tabla 1 se presenta un cuadro comparativo de riqueza en géneros y especies de escarabajos estercoleros hallados en los estudios más relevantes en Ecuador; estos trabajos han utilizado como metodología de recolección específicamente trampas pitfall cebadas con heces humanas y adicionalmente otros cebos como carroña y frutas en descomposición. Según lo anterior, podemos observar que la región amazónica (con sus estribaciones orientales y cordilleras amazónicas) concentran la mayor diversidad de escarabajos estercoleros en Ecuador. Sin embargo, aún faltan estudios locales que permitan una mayor comparación de estos resultados. Este trabajo presenta un número de especies menor que el hallado por Carpio et al. (2009), quienes registraron 69 especies, en bosques de tierra firme amazónicos, y también menor que el registrado por Celi et al. (2004), con 105 especies, realizado en un gradiente altitudinal en bosques siempreverdes de piedemonte, bosques siempre verdes montanos bajos y bosques de neblina montanos, en tres meses de recolección.

Tabla 1. Comparación de estudios publicados sobre escarabajos estercoleros en Ecuador.

\begin{tabular}{|c|c|c|c|c|c|}
\hline Autor (Año) & Localidad-Provincia & $\begin{array}{c}\text { Altitud } \\
\text { (m s. n. m.) }\end{array}$ & $\begin{array}{r}\text { Muestreos } \\
\text { (en meses) }\end{array}$ & Géneros & Especies \\
\hline Peck \& Forsyth (1982) & $\begin{array}{l}\text { Estación Río Palenque-Los Ríos } \\
\text { (costa pacífica) }\end{array}$ & $200-250$ & 3 & 13 & 36 \\
\hline Enríquez \& Onore (2001) & $\begin{array}{l}\text { Otonga-Cotopaxi } \\
\text { (estribación andina) }\end{array}$ & 2000 & 12 & 10 & 14 \\
\hline Celi et al. (2004) & $\begin{array}{l}\text { Angel Rouby y Untsuant } \\
\text { (Kutukú)-Morona Santiago } \\
\text { (cordilleras amazónicas) }\end{array}$ & $500-2000$ & 3 & 17 & 105 \\
\hline Carvajal \& Villamarín (2007) & $\begin{array}{l}\text { B. P. Oglán-Pastaza } \\
\text { (amazonía) }\end{array}$ & 581 & 1 & 16 & 34 \\
\hline Carpio et al. (2009) & $\begin{array}{l}\text { Estación Científica } \\
\text { Chiruisla-Orellana } \\
\text { (amazonía) }\end{array}$ & $180-250$ & 3 & 19 & 69 \\
\hline Domínguez et al. (2015) & $\begin{array}{l}\text { Alamala-Loja } \\
\text { (costa pacífica, } \\
\text { estribación occidental) }\end{array}$ & $390-590$ & 9 & 6 & 6 \\
\hline Espinoza \& Noriega (2018) & $\begin{array}{l}\text { Papallacta, Baeza, Tena y } \\
\text { Misahualli-Napo } \\
\text { (amazonía) }\end{array}$ & $400-2600$ & 2 & 14 & 54 \\
\hline Este estudio & $\begin{array}{l}\text { B. P. Oglán-Pastaza } \\
\text { (amazonía) }\end{array}$ & $540-950$ & 18 & 18 & 65 \\
\hline
\end{tabular}


El número de especies registrado en este estudio es considerablemente mayor (casi el doble) que los valores obtenidos para la misma localidad por Carvajal \& Villamarín (2007), quienes citan 34 especies pertenecientes a 16 géneros. En aquel estudio se realizó solamente un muestreo en dos transectos (uno en bosque maduro y otro en una zona alterada, ambos cercanos a la casa estancia) y 48 horas de recolección, utilizando trampas pitfall cebadas con excremento humano.

Gremios tróficos. Con respecto al grupo funcional, los patrones registrados en este estudio para escarabajos cavadores, rodadores y moradores son similares a los encontrados en otros estudios, especialmente en la Amazonía ecuatoriana y el Neotrópico. Esos estudios muestran un mayor número de especies de escarabajos cavadores, que representan el doble de especies de escarabajos rodadores, y estos últimos representan el doble de especies de escarabajos moradores. Así, en bosques húmedos tropicales, los cavadores están entre 58.33 y $69.57 \%$, los rodadores están entre 23.45 y $33.33 \%$ y los moradores están entre 6.52 y $12.96 \%$ (Klein, 1989; Escobar, 2000; Feer, 2000; Andersen, 2002; Pulido Herrera et al., 2003; Celi et al., 2004; Carpio et al., 2009; Espinoza \& Noriega, 2018). Las especies más dominantes en abundancia para cada grupo funcional fueron: Dichotomius quinquelobatus (Figura 5A, escarabajo cavador de tamaño grande); Deltochilum crenulipes (Figura 4A, escarabajo rodador de tamaño mediano) y Eurysternus caribaeus (Figura 5D, escarabajo morador de tamaño mediano), que coincidencialmente son también las especies más abundantes en esta localidad.

\section{Conclusiones}

Pensamos que la gran mayoría de especies son propias de los bosques de tierras bajas, como Sylvicanthon proseni (Figura 7H); otras de bosques de piedemonte sobre los $500 \mathrm{~m}$ s. n. m., como Dichotomius quinquelobatus (Figura 5A) y algunas otras son transitorias de bosques de menor altitud hacia bosques nublados sobre los 1300 m s. n. m., como Scybalocanthon kaestneri (Figura 7G), por lo que afirmamos que este tipo de ecosistemas ama- zónicos son muy diversos con respecto a otros biomas del Ecuador (ver Tabla 1). Sugerimos realizar estudios de marcaje, captura y recaptura, como el realizado por Cultid et al. (2015) con el objetivo de conocer el tamaño poblacional y las posibles migraciones entre ecosistemas y paisajes.

Adicionalmente con este inventario actualizado y registro fotográfico queremos contribuir a la conservación de la coprofauna y ser un soporte para posibles evaluaciones de la calidad ambiental en Oglán, principalmente por afectaciones futuras a causa de la industria petrolera que pueden empezar en cualquier momento (Baquero, 2006), impactando irreversiblemente a esta comunidad y algunas poblaciones de especies sensibles o endémicas. Lo evidenciado por Carpio et al. (2009), en la localidad de Chriruisla al momento de construir vías de acceso para plataformas petroleras es un ejemplo claro de este problema.

Finalmente, es necesario involucrar a las comunidades indígenas existentes en Oglán sobre temas de monitoreo ambiental y planes de manejo que puedan realizarse con estos escarabajos. En otras regiones de Ecuador han resultado muy exitosas estas alianzas. Como modelo podemos mencionar el trabajo elaborado por Celi \& Dávalos (2001) en el Chocó biogeográfico ecuatoriano, provincia de Esmeraldas, donde se obtuvieron resultados como la elaboración de un manual y guías de identificación de especies que sirvieron para la capacitación y formación de biólogos en comunidades indígenas y afrodescendientes.

\section{Agradecimientos}

Agradecemos de manera muy especial a la comunidad Kichwa Pablo López de Oglán Alto por la logística y amabilidad prestada. Posteriormente al MUTPL y el biólogo Diego Marín-Armijos (Museo y colección de insectos de la Universidad Particular de Loja) por el uso de las fotografías Canthidium aff. gerstackeri y Canthidium sp 2. A Pablo Araujo, Elizabeth Carrillo, Juan Vieira, María de los Ángeles Simbaña, Santiago Andi- 
no y Ernesto Villacrés por su colaboración en el trabajo de campo. Al Dr. Nelson Rodríguez director de la Estación Científica Juri Juri Kawsay, de la Universidad Central del Ecuador por el apoyo y las gestiones realizadas para la logística. A las autoridades de la Escuela de Ciencias Biológicas de la Universidad Central del Ecuador, por la aprobación para la publicación de los datos de la tesis de la coautora Soraya Delgado. A los doctores Ángel Solís y Fernando Z. Vaz-De-Mello por la identificación de parte del material colectado. A la AECID por el financiamiento del proyecto: Consolidación de la Estación Científica Amazónica Juri Juri Kawsay: ligando la investigación y el desarrollo sostenible. Cooperación interuniversitaria e investigación científica entre España e Iberoamérica (BOE 23.07.2007), Código D/7556/07.

\section{Referencias}

Andresen, E. (2002). Dung beetles in a Central Amazonian rainforest and their ecological role as secondary seed disperses. Ecological Entomology, 27, 257-270.

Arnaud, P. (2002). Les Coléoptères du Monde, Vol. 28. Phanaeini. Dendropaemon, Tetramereia, Homalotarsus, Megatharsis, Diabroctis, Coprophanaeus, Oxysternon, Phanaeus, Sulcophanaeus. Canterbury, England: Hillside Books. 151 pp.

Baquero, P. T. (2006). Formulación de la política petrolera. En Fontaine, G. (Ed.). Petróleo y desarrollo sostenible en Ecuador. 3. Las ganancias y pérdidas. Pp. 138153. Ecuador: FLACSO, Friedrich Ebert Stiftung y Petrobras.

Bezdek, A. \& Hajek, J. (2011). Catalogue of type specimens of beetles (Coleoptera) deposited in the National Museum, Prague, Czech Republic. Acta Entomologica Musei Nationalis Pragae, 51(1), 349-378.

Canhedo, V. L. (2006). Revisão taxonômica do gênero Anomiopus Westwood, 1842 (Coleoptera, Scarabaeidae, Scarabaeinae). Archivos de Zoologia, 37, 349-502.

Carpio, C., Donoso, D., Ramón, G. \& Dangles, O. (2009). Short term response of dung beetles communities to disturbance by road construction in the Ecuadorian Amazon. Annales de la Société Entomologique de France, 4, 455-469.
Carvajal, V. \& Villamarín, S. (2007). Diversidad de escarabajos coprófagos (Coleoptera: Scarabaeoidea) en el bosque protector Pablo López del Oglán Alto, Pastaza, Ecuador. Revista Politécnica, 27, 96-10.

Chamorro, W., Marín-Armijos, D., Asenjo, A. \& VazDe-Mello, F. Z. (2019). Scarabaeinae dung beetles from Ecuador: a catalog, nomenclatural acts, and distribution records. ZooKeys, 826, 1-343.

Chamorro, W., Marín-Armijos, D., Granda, V. \& VazDe-Mello, F. Z. (2018). Listado de especies y clave de géneros y subgéneros de escarabajos estercoleros (Coleoptera: Scarabaeidae: Scarabaeinae) presentes y presuntos para Ecuador. Revista Colombiana de Entomología, 44(1), 72-100.

Cook, J. (2002). A revision of the neotropical genus Cryptocanthon Balthasar (Coleoptera: Scarabaeidae: Scarabaeinae). Coleopterists Bulletin, 56, 3-96.

Cook, J. (1998). A revision of the neotropical genus Bdelyrus Harold (Coleoptera: Scarabaeidae: Scarabaeinae). The Canadian Entomologist, 130, 631-689.

Celi, J., Terneus, L., Torres, J. \& Ortega, M. (2004). Dung beetles (Coleoptera: Scarabaeinae) diversity in an altitudinal gradient in the Cutukú range, Morona Santiago, Ecuadorian Amazon. Lyonia, 7(2), 37-52.

Celi, J. \& Dávalos, A. (2001). Manual de monitoreo. Los escarabajos peloteros como indicadores de la calidad ambiental. Quito: EcoCiencia. 71 pp.

Cerón, C. E., Reyes, C. I., Montalvo, C. I. \& Vargas, L. M. (2007). La cuenca Alta del Río Oglán, Pastaza-Ecuador, diversidad, ecología y flora. Quito: Editorial Universitaria, $184 \mathrm{pp}$.

Cultid-Medina, C. A., Martínez-Quintero, B. G., Escobar, F. \& Chacon, P. (2015). Movement and population size of two dung beetle species in an Andean agricultural landscape dominated by sun-grown coffee. Journal of Insect Conservation, 19, 617-626.

Domínguez, D., Marín-Armijos, D. \& Ruíz, C. (2015). Structure of dung beetle communities in an altitudinal gradient of neotropical dry forest. Neotropical Entomology, 44, 40-46.

Edmonds, W. D. \& Zídek, J. (2012). Taxonomy of Phanaeus revisited: Revised keys to and comments on species of the New World dung beetle genus Phanaeus MacLeay, 1819 (Coleoptera: Scarabaeidae: Scarabaeinae: Phanaeini). Insecta Mundi, 274, 1-108. 
Edmonds, W. D. \& Zídek, J. (2010). A taxonomic review of the neotropical genus Coprophanaeus Olsoufieff, 1924 (Coleoptera: Scarabaeidae, Scarabaeinae). Insecta Mundi, 129, 1-111.

Edmonds, W. D. \& Zídek, J. (2004). Revision of the neotropical dung beetle genus Oxysternon (Coleoptera: Scarabaeidae: Scarabaeinae). Folia Heyrovskyana, 11, 1-58.

Enríquez, T. \& Onore, G. (2001). Análisis de la entomofauna copro-necrófaga (Coleoptera: Scarabaeidae: Scarabaeinae) y comparación de su diversidad en tres tipos de hábitats en el Bosque Nublado Otonga. En Nieder, J. \& Barthlott, W. (Eds.). Epiphytes and Canopy Fauna of the Otonga Rain Forest (Ecuador). Pp: 261-274. Bonn: Volkswagen Foundation (2/2).

Erwin, T. (1989). Canopy arthropod biodiversity: a chronology of sampling techniques and results. Revista peruana de Entomologia, 32, 71-77.

Escobar, F. (2000). Diversidad de coleópteros coprófagos (Scarabaeidae: Scarabaeinae) en un mosaico de habitats en la Reserva Natural Nukak, Guaviare, Colombia. Acta Zoológica. Mexicana, 79, 103-121.

Espinoza, V. R. \& Noriega, J. A. (2018). Diversity of the dung beetles (Coleoptera: Scarabaeinae) in an altitudinal gradient in the east slope of los Andes, Napo province, Ecuador. Neotropical Biodiversity, 4(1), 144-150.

Favila, M. E. \& Halffter, G. (1997). The use of indicator groups for measuring biodiveristy as related to community structure and function. Acta Zoológica Mexicana, 72, 1-25.

Feer, F. (2000). Les coleóptères coprophages et necrophages (Scarabaeidae S. Str. et Aphodiidae) de la forêt de Guyane Française: Composition spécifique et structure des pleuplements. Annales de la Société Entomologique de France, 36(1), 29-43.

Génier, F. (2009). Le genre Eurysternus Dalman, 1824 (Scarabaeidae: Scarabaeinae: Oniticellini) révision taxonomique et clés de détermination illustrées. Sofia, Bulgaria: Series Faunistica No. 85, Pensoft ed., 430 pp.

Génier, F. (1996). A revision of the neotropical genus Ontherus Erichson (Coleoptera: Scarabaeidae, Sca- rabaeinae). Memoirs of the Entomological Society of Canada, 170, 1-169.

Halffter, G. \& Edmonds, W. D. (1982). The nesting behavior of dung beetles (Scarabaeinae): an ecological and evolutive approach. México: Instituto de Ecología, $177 \mathrm{pp}$.

Klein, B. C. (1989). Effects of forest fragmentation on dung and carrion beetle communities in Central Amazonia. Ecology, 70(6), 1715-1725.

Krajcik, M. (2012). Checklist of the world Scarabaeoidea. Animma, 5, 1-278.

Palacios, J. \& Ontaneda, G. (2009). Boletín Climatológico Anual, Año 2009. Quito: INAMHI, Instituto Nacional de Metereología e Hidrología.

Palacios, W., Cerón, C. \& Valencia, R. (1999). Las formaciones naturales de la Amazonía del Ecuador. En Sierra, R. (Ed.). Propuesta preliminar de un sistema de clasificación de vegetación para el Ecuador continental. Pp: 109-117. Quito: Proyecto INEFAN/GEF-BIRF y EcoCiencia.

Peck, S. \& Forsyth, A. (1982). Composition, structure and competitive behavior in a guild of Ecuadorian rain forest dung beetles (Coleoptera, Scarabaeidae). Canadian Journal of Zoology, 60, 1624-1634.

Pulido Herrera, L. A., Riveros, R. A., Harders, F. G. \& Hildebrand, P. V. (2003). Escarabajos coprófagos (Coleoptera: Scarabaeidae: Scarabaeinae) del Parque Nacional Natural "Serranía de Chiribiquete", Caqueta, Colombia (Parte I). Escarabeidos de Latinoamerica: Estado del conocimiento, 3, 51-58.

Ratcliffe, B. C. \& Smith, A. B. T. (1999). New species of Canthonella from Amazonian Brazil. The Coleopterists Bulletin, 53(1), 1-7.

Silva, F., Louzada, J. \& Vaz-de-Mello, F. Z. (2015). A revision of the Deltochilum subgenus Aganhyboma Kolbe, 1893 (Coleoptera: Scarabaeidae: Scarabaeinae). Zootaxa, 3925(4), 451-504.

Vaz-de-Mello, F. Z., Edmonds, W. D., Ocampo, F. C. \& Schoolmeesters, P. (2011). A multilingual key to the genera and subgenera of the subfamily Scarabaeinae of the New World (Coleoptera: Scarabaeidae). Zootaxa, 2854, 1-73. 
Anexo 1. Inventario de especies para escarabajos copronecrófagos (Coleoptera: Scarabaeidae: Scarabaeinae) del B. P. Oglán Alto, según las metodologías de muestreo.

Disponible en línea: http://revistas.humboldt.org.co/index.php/biota/rt/suppFiles/573/0.

\section{William R. Chamorro}

(Autor de correspondencia)

Universidad Técnica Particular de Loja,

Museo de Zoología MUTPL,

Departamento de Ciencias Biológicas

Loja, Ecuador

william.chamorro@gmail.com

https://orcid.org/0000-0003-1755-7852

\section{Freddy O. Gallo}

Instituto para Conservación y Capacitación Ambiental (ICCA)

Quito, Ecuador

fviracocha@hotmail.com

https://orcid.org/0000-0002-0480-2348

\section{Soraya Delgado}

Universidad Central del Ecuador,

Facultad de Ciencias Médicas por Facultad de Ciencias Biológicas Quito, Ecuador

soraya_delgadotipan@yahoo.es

https://orcid.org/0000-0002-4336-4686

\section{Sandra I. Enríquez}

Universidad Central del Ecuador,

Instituto de Investigación en Salud Pública y Zoonosis (CIZ)

Quito, Ecuador

ienriquez@uce.edu.ec

https://orcid.org/0000-0003-3501-0076

\section{Verónica Guasumba}

ENTRIX Consulting Group

Tumbaco, Ecuador

veritos_g@hotmail.com

https://orcid.org/0000-0002-7186-088X

\section{Germán López-Iborra}

Universidad de Alicante

Instituto Multidisciplinar para el estudio del medio "Ramón Margalef"

Alicante, España

german.lopeziborra5@gmail.com

https://orcid.org/0000-0003-3045-5498
Los escarabajos estercoleros (Coleoptera: Scarabaeidae: Scarabaeinae) del Bosque Protector Oglán Alto, Pastaza, Ecuador

Citación del artículo: Chamorro, W. R., Gallo, F. O., Delgado, S., Enríquez, S. I., Guasumba, V. \& López-Iborra, G. (2019). Los escarabajos estercoleros (Coleoptera: Scarabaeidae: Scarabaeinae) del Bosque Protector Oglán Alto, Pastaza, Ecuador. Biota Colombiana, 20(1), 34-49. DOI: 10.21068/c2019.v20n01a03.

Recibido: 23 de agosto de 2018

Aceptado: 25 de enero de 2019 